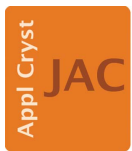

JOURNAL OF

APPLIED CRYSTALLOGRAPHY

ISSN 1600-5767

Received 11 September 2020

Accepted 8 March 2021

Edited by G. J. McIntyre, Australian Nuclear Science and Technology Organisation, Lucas Heights, Australia

Keywords: quantum crystallography; Hirshfeld atom refinement; X-ray constrained wavefunction fitting.

CCDC references: 1987830; 2027443; $2027444 ; 2027445 ; 2027446$

Supporting information: this article has supporting information at journals.iucr.org/j

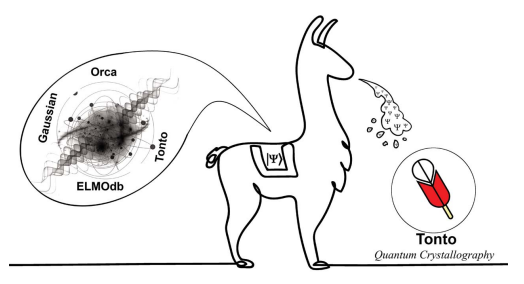
OPEN $\odot$ ACCESS

\section{lamaGOET: an interface for quantum crystallography}

\author{
Lorraine A. Malaspina, ${ }^{\mathrm{a}, \mathrm{b} *}$ Alessandro Genoni ${ }^{\mathrm{c}}$ and Simon Grabowsky ${ }^{\mathrm{a}, \mathrm{b} *}$ \\ a Universität Bern, Departement für Chemie, Biochemie und Pharmazie, Freiestrasse 3, 3012 Bern, Switzerland, \\ ${ }^{b}$ Universität Bremen, Fachbereich 2 - Biologie/Chemie, Institut für Anorganische Chemie und Kristallographie, Leobener \\ Strasse 3, 28359 Bremen, Germany, and ' Université de Lorraine and CNRS, Laboratoire de Physique et Chimie \\ Théoriques (LPCT), UMR CNRS 7019, 1 Boulevard Arago, 57078 Metz, France. *Correspondence e-mail: \\ lorraine.malaspina@dcb.unibe.ch, simon.grabowsky@dcb.unibe.ch
}

In quantum crystallography, theoretical calculations and crystallographic refinements are closely intertwined. This means that the employed software must be able to perform both quantum-mechanical calculations and crystallographic least-squares refinements. So far, the program Tonto is the only one able to do that. The lamaGOET interface described herein deals with this issue since it interfaces dedicated quantum-chemical software (the widely used Gaussian package and the specialized ELMOdb program) with the refinement capabilities of Tonto. Three different flavours of quantum-crystallographic refinements of the dipetide glycyl-L-threonine dihydrate are presented to showcase the capabilities of lamaGOET: Hirshfeld atom refinement (HAR), HAR-ELMO, namely HAR coupled with extremely localized molecular orbitals, and X-ray constrained wavefunction fitting.

\section{Introduction}

An accurate determination of the electronic structure of a compound allows the derivation of many properties related, for example, to its reactivity or stability. One way of obtaining this information is through the theoretical calculation of a wavefunction for the compound under investigation. Wavefunctions are mathematical objects that intrinsically contain all the information of quantum-mechanical systems in specific pure states, most often the ground electronic state. Here, we are concerned with the electronic wavefunction, as the square of the electronic wavefunction is related to the electron density. Nowadays, with increasing computational power and the continuous development of sophisticated methods, many different software programs for calculation of wavefunctions are available, e.g. Quantum ESPRESSO (Giannozzi et al., 2017), Turbomole (Furche et al., 2014), Crystal (Dovesi et al., 2018), Gaussian (Frisch et al., 2016), Orca (Neese, 2012), Tonto (Jayatilaka \& Grimwood, 2003) and many more.

A reconstruction of the electron density can also be achieved experimentally, e.g. from scattering experiments such as single-crystal X-ray diffraction. However, reconstructing the electron density of crystal structures always requires theoretical models to interpret the measured data, hence intrinsically connecting crystallography and quantum mechanics (Genoni et al., 2018; Korlyukov \& Nelyubina, 2019). The vast majority of crystal structure refinements use the independent atom model (IAM), where every atom is represented as a theoretically calculated spherical noninteracting averaged ground-state electron density (Compton, 1915; Sheldrick, 2008). This model ignores any deformation of 
electron density that is due to lone-pair regions, primary chemical bonding (covalent, metallic, ionic) and secondary interactions (e.g. hydrogen bonding, dipole-dipole interactions and London dispersion). There are electron-density models more accurate than the IAM that account for the nonsphericity of the atomic electron distributions (Koritsanszky \& Coppens, 2001).

Multipole models (MMs) have been designed specifically to model chemical-bonding effects (Dawson, 1967; KurkiSuonio, 1968; Hirshfeld, 1971; Stewart, 1976; Coppens, 2005). The most widely used MM variant is based on the HansenCoppens pseudoatom formalism (Hansen \& Coppens, 1978; Coppens, 1997), where each atom is modelled by a superposition of radial and spherical harmonic functions. Atomic scattering factors are retrieved from a combination of tabulated spherical contributions and refined multipole parameters. This means that in multipole modelling both the molecular geometry, including atomic displacement parameters, and electron-density parameters are obtained by refinement against the measured structure factors. Alternatively, multipole parameters can be transferred from databanks (either constructed from theoretical calculations or averaged over experimental multipole refinements) and fixed during the refinement of positions and anisotropic displacement parameters (Dittrich et al., 2005; Dadda et al., 2012; Bạk et al., 2011). By virtue of their construction, multipole databanks are suitable for the refinement of peptide and protein crystal structures with nonspherical atomic form factors (Jelsch et al., 2000; Dittrich et al., 2010).

Beyond multipole modelling, there are methods that make direct use of quantum-mechanical wavefunctions to model experimental diffraction data by taking into account atomic nonsphericity, and these are discussed in the following paragraphs. These methods belong to the emerging field of quantum crystallography (QCr) (Grabowsky et al., 2017, 2020; Genoni et al., 2018). The majority of QCr methods have to date been exclusively implemented and run in the software Tonto (Jayatilaka \& Grimwood, 2003). The software lamaGOET presented in this work is a graphical user interface (GUI) for Tonto to make its full capability more easily accessible. Therefore, lamaGOET acts as an interface for the three quantum-crystallographic methods described below.

Hirshfeld atom refinement (HAR). HAR (Jayatilaka \& Dittrich, 2008; Capelli et al., 2014) is an established method for modelling X-ray diffraction data with the help of nonspherical atomic scattering factors. In HAR, quantum-mechanical calculations are used to derive the theoretical electron density of the molecule under investigation. From this quantummechanical electron density, nonspherical atomic scattering factors, which are used in the refinement of the experimental data, are obtained using Hirshfeld's stockholder partitioning of the electron density (Hirshfeld, 1977a,b). The following steps are performed during HAR:

(1) A single point energy computation provides an electron density distribution, using the current geometric parameters.

(2) The obtained electron density is then Hirshfeld partitioned into atomic electron-density functions (the Hirshfeld atoms), which are afterwards Fourier transformed to provide tailor-made nonspherical atomic scattering factors for the system under investigation.

(3) A least-squares refinement of positional and displacement parameters is carried out using the nonspherical scattering factors obtained in the previous step.

These steps are repeated until full convergence is achieved in energy and geometric parameters. The atomic scattering factors are purely theoretical, and only the atomic coordinates and the displacement parameters are refined against the experimental data. It has been shown that HAR is able to generate from $\mathrm{X}$-ray data bond distances involving $\mathrm{H}$ atoms that are as accurate and precise as those obtained from neutron-diffraction studies (Woińska et al., 2016; Fugel et al., 2018; Sanjuan-Szklarz et al., 2020), thus overcoming the limitations of IAM and MM in the determination of $\mathrm{H}$-atom positions.

Through lamaGOET, HAR can be performed on the basis of wavefunctions calculated with the Gaussian software (Frisch et al., 2016). In other words, lamaGOET allows interfacing Tonto and Gaussian directly. This gives access to quantum-mechanical methods otherwise not available, without detriment of any functionality in the original software Tonto.

HAR-ELMO. A limitation of HAR is the fact that it requires a Hartree-Fock (HF) or density-functional-theory (DFT) computation before each refinement step, so that it is computationally expensive. Hence, it cannot be readily applied to larger systems such as macromolecules or compounds containing heavy elements. However, Meyer \& Genoni (2018) have recently constructed a library of extremely localized molecular orbitals (ELMOs). These molecular orbitals are strictly localized on small molecular units, i.e. atoms, bonds and functional groups. For this reason, they are easily transferable from molecule to molecule (Meyer, Guillot, Ruiz-Lopez \& Genoni, 2016; Meyer, Guillot, Ruiz-Lopez, Jelsch \& Genoni, 2016), allowing the quick reconstruction of wavefunctions and electron densities of proteins through instantaneous transfer of ELMOs from the databank with the software ELMOdb (Meyer \& Genoni, 2018). At present, the databank includes ELMOs for all the naturally encoded amino acids. Therefore, for coordination compounds and corresponding ligands, ELMOs have to be calculated once before the transfer. It was recently demonstrated that the new HARELMO method allows one to perform refinements that produce $\mathrm{H}$-atom parameters as accurate and precise as those resulting from neutron diffraction or original HAR for small molecules (Malaspina et al., 2019).

For HAR-ELMO applications, lamaGOET interfaces the ELMOdb software (Meyer \& Genoni, 2018) with Tonto (Malaspina et al., 2019). The ELMOdb software takes care of the rapid generation of wavefunctions from ELMO building blocks, lamaGOET transfers these wavefunctions to Tonto, and Tonto carries out the Hirshfeld atom partitioning and crystallographic least-squares refinement. Although other fragment approaches have recently been developed (Zheng et al., 2020; Bergmann et al., 2020), to the best of our knowledge, 
HAR-ELMO within lamaGOET is currently the only available method that has been used to refine a protein with quantum crystallographically derived nonspherical atomic scattering factors beyond multipole database techniques (Malaspina et al., 2019).

$X$-ray constrained wavefunction $(X C W)$ fitting. XCW fitting (Jayatilaka, 1998; Jayatilaka \& Grimwood, 2001; Grimwood \& Jayatilaka, 2001) allows for the fitting of molecular orbital coefficients to measured structure factors. Whereas HAR and HAR-ELMO as well as multipole database techniques derive nonspherical atomic form factors theoretically and refine only coordinates and displacement parameters, in the XCW strategy the electron density is refined via the fitting of molecular orbitals. This allows access to experimentally restrained wavefunctions.

A purely theoretical wavefunction is initially used as ansatz for the determination of the fitted wavefunction. In this procedure, instead of minimizing only the energy of the system under examination in a self-consistent field calculation, a new functional $L[\mathbf{c}]$ is minimized, which is the sum of the energy of the system $E[\mathbf{c}]$ and a term that represents the restraints given by the experimental X-ray diffraction data:

$$
L[\mathbf{c}]=E[\mathbf{c}]+\lambda\left(\chi^{2}[\mathbf{c}]-\Delta\right) .
$$

c is the matrix of the molecular orbital coefficients that are fitted to the experimental structure factors during the calculation, $\lambda$ is an external multiplier that is manually adjusted during the computation and gives the strength of the experimental restraints, $\chi^{2}$ is a measure of the statistical agreement between experimental and theoretical structure factors, and $\Delta$ is the desired agreement between experimental and computed values. Therefore, in XCW fitting, experimental information is embedded into the theoretical wavefunction, in order to obtain the best possible description of the electron density.

During this procedure, all geometric parameters are unaffected. Therefore it is advisable to perform XCW fitting in the best possible derived geometry. The usage of XCW fitting after HAR is defined as X-ray wavefunction refinement (XWR) (Woińska et al., 2017). lamaGOET can set up very specialized input files for XWR and XCW fitting procedures.

A large number of studies have shown that XCW fitting allows acquisition of reliable charge-density distributions for determination of material properties (Whitten et al., 2006; Jayatilaka et al., 2009; Hickstein et al., 2013; Cole \& Hickstein, 2013) as well as capturing polarization and crystal-field effects (Grabowsky et al., 2020; Ernst et al., 2020). Bytheway et al. (2007) and Bučinský et al. (2016) have also investigated theoretically what the detectability likelihood of electron correlation in diffraction data is, and Genoni et al. (2017) have proven that XCW fitting is in principle able to capture electron correlation to a certain extent.

In addition to the chemical example of glycyl-L-threonine dihydrate (Benabicha et al., 2000) related to the three QCr methods described above, in this paper we also briefly discuss the scope of generating plots of various properties with lamaGOET, and how lamaGOET takes self-consistent
Hirshfeld cluster charges from Tonto to perturb molecular wavefunctions in Gaussian geometry optimizations.

\section{2. lamaGOET: platform, availability and use}

Traditionally, a myriad of different stand-alone utilities have been used by the crystallographic community, mostly in the Fortran programming language. However, more importantly, most crystallographic software can be run using a commandline interpreter. Therefore, interfacing different software can be easily achieved in command language. The lamaGOET interface started as a small bash script to perform a specific job, namely a HAR using Gaussian for the SCF calculations and Tonto for the refinements. Its utilities and functions rapidly increased, making it a tool for broad use in quantum crystallography. The latest version of lamaGOET is still written in bash, which makes it easy for users to read and understand the code. This also allows easy transferability across different operating systems. The script can be run on Linux and MacOS platforms using the native command-line interpreter. The prerequisites to run lamaGOET are usually default in any bash interpreter. These are gawk, zenity and coreutils. The increasing number of features and options led to the implementation of the graphical interface using gtkdialog, which is a GUI-creation utility that can be used with an arbitrary interpreter. By running the installation script provided with lamaGOET, all these dependencies are automatically installed, including gtkdialog. The lamaGOET script encourages code reuse and distribution and is subject to the GNU Public License. lamaGOET was written by LAM (lamaGOET $=$ Lorraine A. Malaspina Gaussian Orca ELMO Tonto) (Malaspina, 2020) and can be obtained free of charge at http://www.tinyurl.com/lamaGOET (source code is also available at https://github.com/lomalaspina/lamaGOET).

Once the installation script has been run successfully, the GUI can be called by typing lamaGOET inside the chosen command-line interpreter from the folder where the result files are to be written. As input, a file which contains the initial geometry and crystal information of the structure is needed; this can be a CIF or a Protein Data Bank (PDB) file if the job uses the ELMO libraries. In addition, a reflection file in free format is needed as input for all quantum-crystallographic treatments. A flowchart visualizing the way in which lamaGOET enables HAR and HAR-ELMO by interfacing different quantum-mechanical software with Tonto is given in Fig. 1(a). Moreover, the procedure of an XCW fitting with lamaGOET is visualized in Fig. 1(b). Full explanations of all available functions and options to be chosen for the quantumcrystallographic examples discussed in Section 3 are given in the supporting information (Section 1).

There are a few special versions of lamaGOET. In Windows, it can be run using different available $\mathrm{X}$ servers or the GNU environment. It has been successfully tested on Windows platforms using the MinGW, MobaXterm and Cygwin tools. For use on computer clusters or supercomputers, two separate scripts are available for download. The first contains the graphical user interface (called GUI-lamaGOET), 


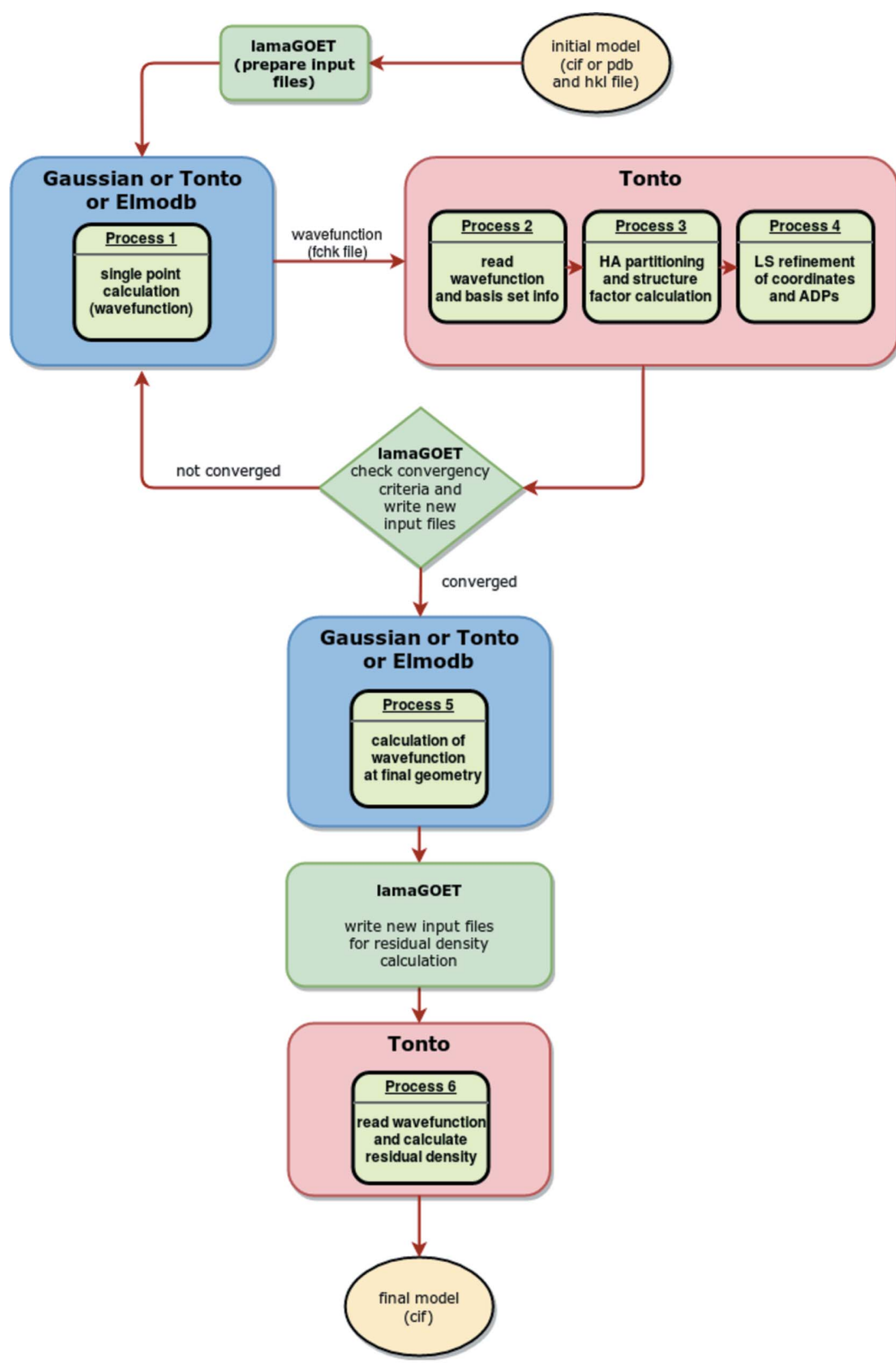

(a)

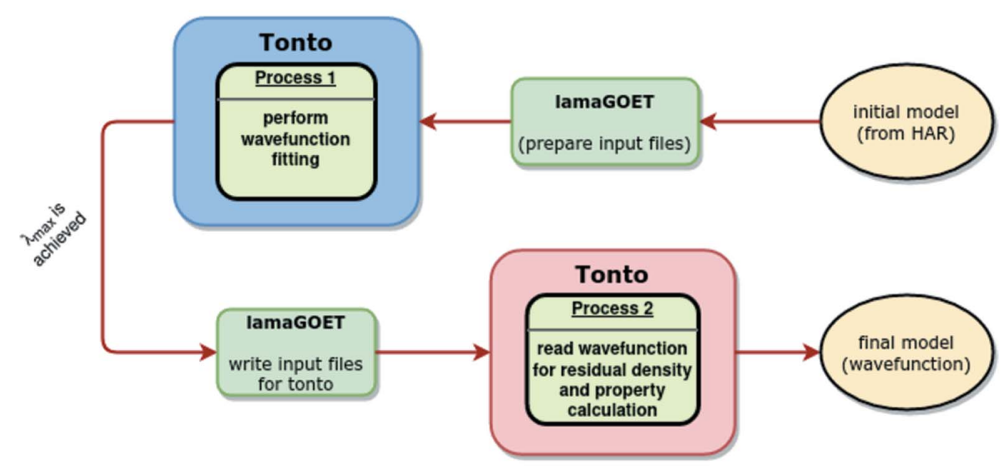

(b)

Figure 1

(a) Flowchart visualizing the procedure for HAR or HAR-ELMO controlled by lamaGOET, interfacing Tonto for the Hirshfeld stockholder partitioning and refinement with other quantum-mechanical software for the wavefunction calculation. (b) Procedure of the XCW fitting in Tonto controlled by lamaGOET. which should be placed on the user's local machine. The second reads the inputs provided in the GUI and runs the script (RUN-lamaGOET). It should be placed on the computer cluster. Versions using the Torque (PBS) and the YARN (through spark.cmd) queuing systems are available at the github page https:/github.com/ lomalaspina/lamaGOET.

Tonto is available at https:/github.com/dylanjayatilaka/tonto. The github page provides stepby-step tutorials on how to install and compile Tonto for all different operating systems. We recommend to use gfortran- 8 for the Tonto compilation on a Linux system.

$E L M O d b$ is a stand-alone program (Meyer \& Genoni, 2018) independent of lamaGOET. It allows the automatic transfer of ELMOs from the available ELMO databanks (Meyer \& Genoni, 2018) to target polypeptide/protein structures. It requires a PDB file as input, which is analysed by the program one residue at a time. For each residue, ELMOdb processes every single fragment by retrieving the orbitals to be transferred and by defining the atomic triads that are necessary to define the matrices for the rotation/ transfer of the ELMOs to the target structure (Meyer, Guillot, Ruiz-Lopez \& Genoni, 2016; Meyer, Guillot, Ruiz-Lopez, Jelsch \& Genoni, 2016; Meyer \& Genoni, 2018). The current version of the ELMO libraries covers all the possible fragments for the 20 naturally encoded amino acids in all their possible protonation states and forms (N-terminal, C-terminal and nonterminal) and the water molecule. The stored ELMOs are available in different standard basis sets of quantum chemistry [6-31G, 6-31G $(d, p)$, 6-311G, 6-311G $(d, p)$ and cc-pVDZ]. In addition, $E L M O d b$ has the option of reading customized ELMOs expanded on any basis set and corresponding to particular fragments, ligands or solvent molecules that may constitute the systems under examination. These tailor-made ELMOs must be preliminarily computed on appropriate model molecules and then stored in a suitable directory where the program can retrieve them when necessary. ELMOdb finally provides an output file with general information on the performed computation, along with a binary file for the final rotated ELMOs, a binary file incorporating the associated ELMO one-electron density matrix, and a Gaussian-formatted checkpoint file that is used to perform subsequent analyses or calculations. The ELMOdb program and the ELMO libraries are currently available free of charge by sending a request to the main developer of the software (Alessandro.Genoni@ univ-lorraine.fr) (Meyer \& Genoni, 2018). In the 
course of a HAR-ELMO treatment (Fig. 1), lamaGOET reads the initial or iteratively refined geometry and passes it to $E L M O d b$ for the automatic transfer and rotation of molecular orbitals, together with information on tailor-made residues (if present). lamaGOET then reads the formatted checkpoint file output from ELMOdb and passes it on to Tonto for the leastsquares (LS) refinement.

The lamaGOET interface also facilitates the generation of grid files in Gaussian cube-file format within Tonto for different properties. These grid files are generated from the resulting binary wavefunction files written by Tonto. These binary files are generated through lamaGOET regardless of the software selected for the wavefunction-calculation step. At the moment, all Tonto-generated grid files will contain only atoms of the asymmetric unit which are within the unit-cell dimensions. Therefore, in many cases, pieces of molecules will be omitted for the calculation of cubes. To avoid this problem, lamaGOET offers the user the option to set the size, origin and orientation of the grid file manually. The next paragraph illustrates a short example of properties that can be calculated and plotted; further instructions on how to use this option can be found in the supporting information (at the end of Section 2 and in Figs. S5 and S9).

In the ammonia crystal structure, only a third of the molecule is symmetry independent. We performed HAR of ammonia based on experimental data taken from Boese et al. (1997). We used Tonto at the HF/def2-TZVP level of theory with the option to auto-complete the structure as described in the supporting information. The corresponding CIF is deposited with the Cambridge Structural Database (CSD) under CCDC deposition No. 1987830 and is also provided as supporting information to this article. Fig. 2 shows the related representations of the deformation density distribution, of the distribution of the negative Laplacian of the electron density and of the Becke 88 exchange-correlation potential based on the Kohn-Sham orbitals calculated in the final HAR geometry. In the deformation density and the negative Laplacian, valence-shell charge concentrations (purple, blue) signify bonding and non-bonding effects, here the covalent $\mathrm{N}-\mathrm{H}$ bonds and the nitrogen lone pair, respectively. The exchange-correlation potential is less structured, but it does show the presence of the nitrogen lone pair by a deviation from sphericity into the lone-pair direction.

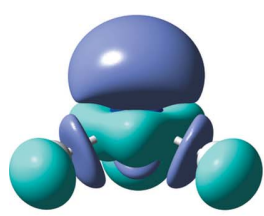

(a)

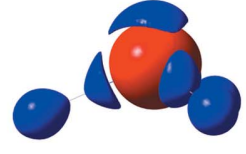

(b)

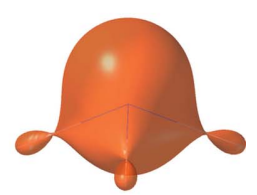

(c)
Figure 2

Different representations of bonding in ammonia; grid files defined with lamaGOET and calculated with Tonto. (a) Deformation density, isosurface at $0.016 \mathrm{e}^{-3}$, purple $=$ positive, turquoise $=$ negative. $($ b) Negative Laplacian of the electron density, isosurface at $35.7 \mathrm{e} \AA^{-5}$, blue $=$ positive, red $=$ negative. $(c)$ Becke88 exchange-correlation potential at 0.75 Hartree $\mathrm{e}^{-1}$. Graphics produced with the software GaussView (Dennington et al., 2008).
Table 1

Crystallographic and measurement details.

\begin{tabular}{ll}
\hline Compound & Glycyl-L-threonine dihydrate \\
\hline Chemical formula & $\mathrm{C}_{6} \mathrm{H}_{12} \mathrm{~N}_{2} \mathrm{O}_{4} \cdot 2 \mathrm{H}_{2} \mathrm{O}$ \\
Formula weight $\left(\mathrm{g} \mathrm{mol}^{-1}\right)$ & 212.21 \\
Crystal size $\left(\mathrm{mm}^{3}\right)$ & $0.300 \times 0.300 \times 0.150$ \\
Crystal habit & Rectangular prism \\
Crystal colour & Colourless \\
Temperature $(\mathrm{K})$ & $110(5)$ \\
Wavelength $(\AA)$ & 0.71068 \\
& \\
Unit cell & \\
$a(\AA)$ & $9.572(3)$ \\
$b(\AA)$ & $10.039(3)$ \\
$c(\AA)$ & $10.548(3)$ \\
Volume $\left(\AA^{3}\right)$ & $1013.7(5)$ \\
Crystal class $/ Z$ & Orthorhombic/4 \\
Space group & $P 2_{1} 2_{1} 2_{1}$ \\
& 15903 \\
No. of reflections & 0.0233 \\
$R_{\text {int }}$ & 5417 \\
Unique reflections & 4579 \\
Unique observed $[F / \sigma(F)>3]$ & $54.91(d=0.44 \AA)$ \\
Reflections $\theta_{\text {max }}\left({ }^{\circ}\right)$ &
\end{tabular}

Although unrelated to QCr, another option available inside the lamaGOET GUI is the possibility of setting up theoretical geometry optimizations of structures in Gaussian using a field of self-consistent Hirshfeld point charges within a defined cluster radius. Unlike the previous steps, this is a fully theoretical approach that allows the user to perform isolatedmolecule optimizations with the influence of the environment. The idea is similar to that of the software baerlauch (Dittrich, Pfitzenreuter \& Hübschle, 2012), where crystal structures are used to provide input files consisting of explicit clusters of molecules for Gaussian optimizations, but in lamaGOET the environment is considered implicitly via symmetry-generated cluster charges, not explicitly as in baerlauch. Geometry optimizations based on such simulations of the environment require significantly less computer power than a fully periodic calculation using the software described in the first paragraph of the Introduction. An example of this lamaGOET option is discussed in Section 3 of the supporting information.

\section{Illustrative scientific example}

A high-resolution charge-density-quality data set of the dipeptide glycyl-L-threonine dihydrate was taken from the literature (Benabicha et al., 2000). Some crystallographic and measurement details are repeated in Table 1. Two different HARs were performed on the available data: (i) HAR-ELMO with lamaGOET, ELMOdb and Tonto utilizing ELMOs expanded on the 6-311G $(d, p)$ basis set, and (ii) a HAR with lamaGOET, Gaussian and Tonto (referred to as 'GaussianHAR') working with a wavefunction obtained at the B3PW91/ def2-TZVP level of theory. The HAR-ELMO treatment is quick (13 min) and does not include any simulation of the crystal field. The Gaussian-HAR is more than seven times slower (94 min) but putatively more accurate, with a DFT functional only accessible via Gaussian (not available for 
Table 2

Refinement statistics for glycyl-L-threonine dihydrate.

\begin{tabular}{|c|c|c|c|c|}
\hline \multirow[b]{2}{*}{ Refinement model } & \multicolumn{2}{|l|}{ HAR } & \multicolumn{2}{|c|}{$\mathrm{XCW}$ fitting based on } \\
\hline & $\begin{array}{l}\text { HAR- } \\
\text { ELMO }\end{array}$ & $\begin{array}{l}\text { Gaussian- } \\
\text { HAR }\end{array}$ & $\begin{array}{l}\text { HAR- } \\
\text { ELMO }\end{array}$ & $\begin{array}{l}\text { Gaussian- } \\
\text { HAR }\end{array}$ \\
\hline No. of parameters & 271 & 271 & 1 & 1 \\
\hline No. of unique observations & 4579 & 4579 & 4579 & 4579 \\
\hline$R$ factor (obs) & 0.029 & 0.029 & 0.027 & 0.026 \\
\hline$w R$ factor (obs) & 0.024 & 0.024 & 0.022 & 0.021 \\
\hline$\chi^{2}$ & 0.814 & 0.781 & 0.639 & 0.581 \\
\hline$\hat{\lambda}_{\max }$ & N/A & N/A & 3.000 & 2.800 \\
\hline Residual density max $\left(\mathrm{e} \AA^{-3}\right)$ & 0.312 & 0.315 & 0.315 & 0.340 \\
\hline Residual density min $\left(\mathrm{e}^{\circ} \AA^{-3}\right)$ & -0.319 & -0.337 & -0.285 & -0.278 \\
\hline Residual density mean $\left(\mathrm{e} \AA^{-3}\right)$ & 0.029 & 0.029 & 0.029 & 0.035 \\
\hline Time of the refinement $(\mathrm{min})$ & 13 & 94 & N/A & N/A \\
\hline CCDC deposition No. & 2027445 & 2027443 & 2027446 & 2027444 \\
\hline
\end{tabular}

ELMO generation or in Tonto) and a cluster of point charges around the asymmetric unit simulating the crystalfield effect. The refinement statistics are summarized in Table 2 and the molecular geometries with anisotropic displacement parameters (ADPs) for all atoms including $\mathrm{H}$ atoms are shown in Fig. 3. The corresponding CIFs are deposited with the CSD under deposition numbers 2027443, 2027444, 2027445 and
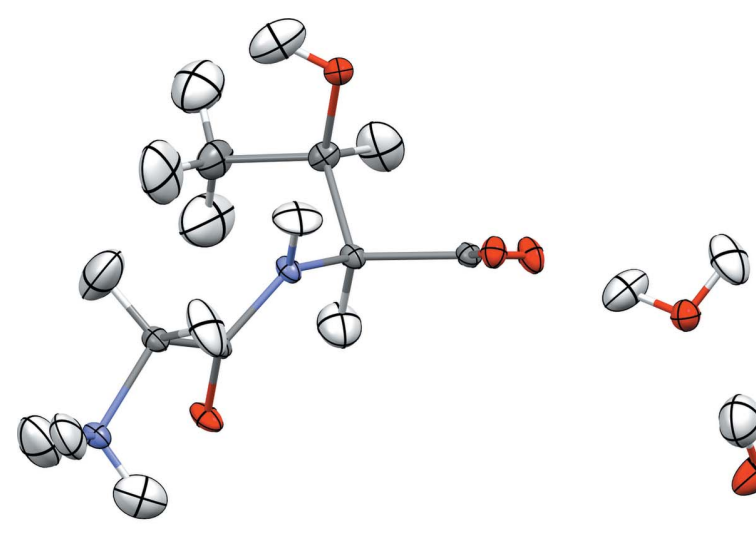

(a)
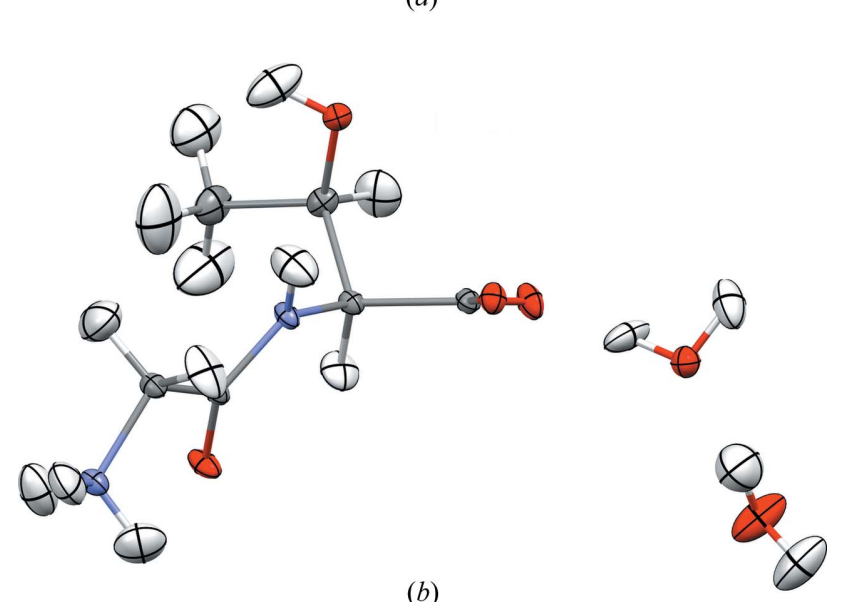

(b)

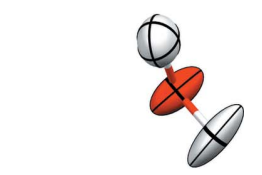

Figure 3

Structure of glycyl-L-threonine dihydrate obtained by (a) HAR-ELMO and $(b)$ Gaussian-HAR. All ADPs are shown at the $50 \%$ probability level. Graphics produced with the software Mercury (Macrae et al., 2020).
2027446 and are also provided as supporting information to this article.

Fig. 4 visualizes the assumptions and approximations that are used in HAR-ELMO and Gaussian-HAR at the level of the electron density. Fig. 4(a) compares the transferredELMO wavefunction with a full Hartree-Fock wavefunction using the same basis set. The differences are systematic: only valence electron density is affected; bonding regions show negative difference density, and nitrogen and oxygen lone-pair regions positive difference density. This means that the extreme localization scheme used in HAR-ELMO underestimates the charge delocalization from lone pairs into bonding regions, which is especially pronounced for the resonance acting in the peptide and carboxylate functional groups.

Whereas Fig. 4(a) visualizes a methodological shortcoming in HAR-ELMO, Fig. 4(b) visualizes a methodological improvement in Gaussian-HAR when DFT and cluster

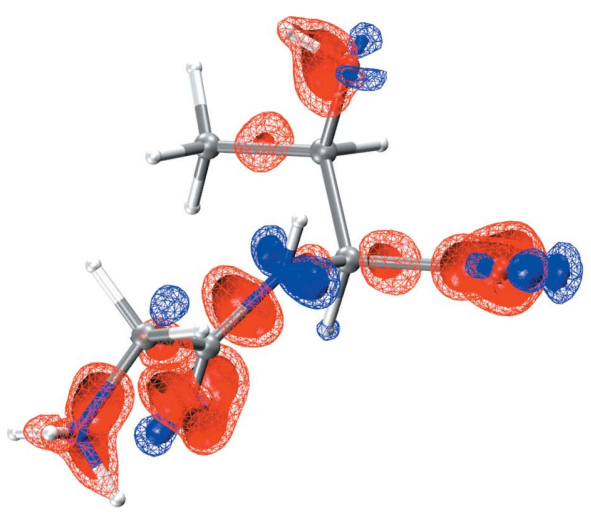

(a)
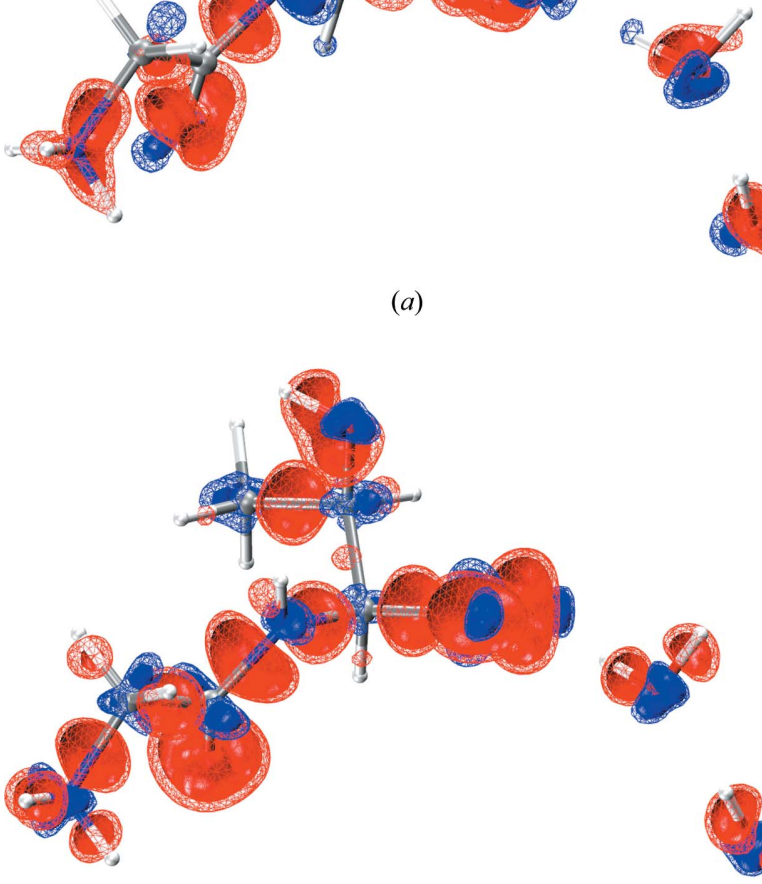

(b)

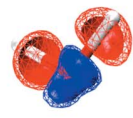

Figure 4

Difference electron density maps for $(a)$ the transferred-ELMO wavefunction $[6-311 \mathrm{G}(d, p)$ basis set] minus the $\operatorname{HF} / 6-311 \mathrm{G}(d, p)$ wavefunction, and for $(b)$ the B3PW91/def2-TZVP wavefunction surrounded by a cluster of point charges minus the HF/def2-TZVP wavefunction without a simulated crystal environment in glycyl-Lthreonine dihydrate. Positive is blue, negative is red. The isovalue of

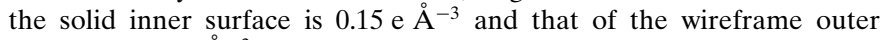
surface is $0.10 \mathrm{e}^{-3}$. The images were generated with the software $V M D$ (Humphrey et al., 1996). 
charges are used instead of an isolated HF wavefunction. This means that Fig. 4(b) describes the combined effect of electron correlation (in the density-functional-theory approximation) plus polarization due to the crystal electric field (approximated by using Hirshfeld point charges) (Dittrich, Sze et al., 2012; Kleemiss, Wieduwilt et al., 2021). Overall, the combined correlation-polarization effect reduces electron density in the valence region and increases it in the atomic cores.

Despite the described differences in the underlying electron densities, the differences between the results of the fast but approximate HAR-ELMO and the slow but higher-level Gaussian-HAR are marginal. The figures of merit for both refinements in Table 2 are nearly the same. Only in the $\chi^{2}$ value does the Gaussian-HAR show a lower value, indicating a slightly better agreement between the model and the measured data. The freely refined hydrogen ADPs visualized in Fig. 3 appear to be physically meaningful in both models. The average $\mathrm{C}-\mathrm{H}$ bond distances agree exactly between the

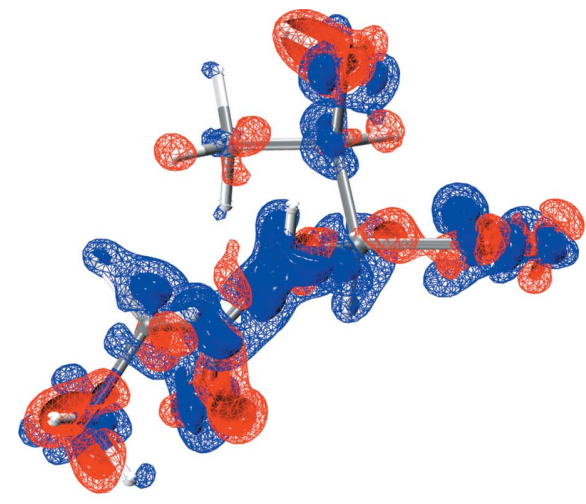

(a)

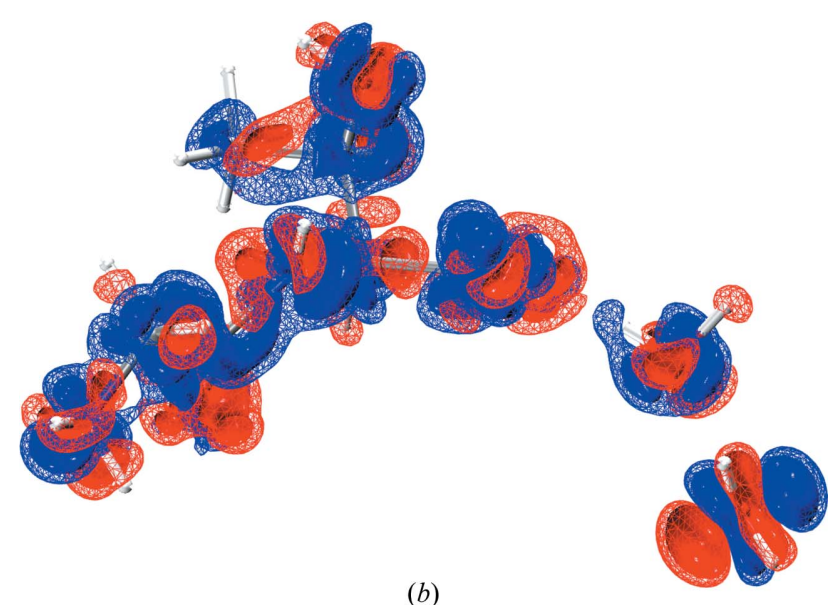

(b)

Figure 5

Difference electron density maps for $(a)$ the XCW fitted wavefunction $(\lambda=3.0)$ minus the non-fitted wavefunction $(\lambda=0.0)$ at the level of theory $\mathrm{HF} / 6-311 \mathrm{G}(d, p)$ and with the geometry from the HAR-ELMO treatment, and $(b)$ the XCW fitted wavefunction $(\lambda=2.8)$ minus the non-fitted wavefunction $(\lambda=0.0)$ at the level of theory HF/def2-TZVP and with the geometry from the Gaussian-HAR treatment in glycyl-Lthreonine dihydrate. Positive is blue, negative is red. The isovalue of the solid inner surface is $0.10 \mathrm{e} \AA^{-3}$ and that of the wireframe outer surface is $0.05 \mathrm{e}^{-3}$. The images were generated with the software $V M D$ (Humphrey et al., 1996). two HAR models, but the $\mathrm{O}-\mathrm{H}$ and $\mathrm{N}-\mathrm{H}$ bonds are on average $0.01 \AA$ longer in the Gaussian-HAR than in HARELMO, and thus closer to reference values from neutron diffraction (Allen \& Bruno, 2010). This is caused by the use of cluster charges in the Gaussian-HAR, whereas in HARELMO the crystal environment is not accounted for (Fugel et al., 2018). Notwithstanding this small advantage, the HARELMO option in lamaGOET produces fast and reliable results for peptides and might be an option for future quantum-crystallographic refinement of protein crystal structures (Malaspina et al., 2019).

Starting from the two slightly different geometries after HAR-ELMO and Gaussian-HAR treatments, two lama$G O E T$-mediated XCW fittings were performed at HF level, each of them without a surrounding cluster of charges. The reason for this choice is to test whether the electron correlation and polarization effects extracted through $\mathrm{XCW}$ fitting from measured structure factors are qualitatively and quantitatively comparable to those associated with the DFT and cluster charge approximations shown in Fig. 4, as well as to those reported in recent papers by Genoni et al. (2017) and Ernst et al. (2020).

Fig. 5 shows that the XCW fitting effects on the electron density are qualitatively similar to the DFT and cluster-charge approximations visualized in Fig. 4, but they are smaller, as shown by the smaller isovalues used. There are two different reasons for this. (i) It has been shown that the XCW fitting effect depends strongly on the resolution of the data set and on the value of the external multiplier $\lambda$ for fitting both electron correlation (Genoni et al., 2017) and polarization (Ernst et al., 2020). Here, a high-resolution data set is used ( $d=$ $0.44 \AA$ ) and a relatively small value of $\lambda_{\max }(2.8 / 3.0)$ was reached before convergence of the calculations ceased. (ii) The effect of electron correlation is overestimated by using a hybrid DFT functional (Medvedev et al., 2017). In addition, the effect of polarization is overestimated by using selfconsistent Hirshfeld charges (Kleemiss, Wieduwilt et al., 2021). In summary, this means that the true effect of electron correlation and polarization on the electron density lies between Figs. 4(b) and 5.

\section{Conclusions}

In this paper, we have demonstrated the usefulness and capabilities of the quantum-crystallographic interface lamaGOET. It facilitates Hirshfeld atom refinement, HAR-ELMO, $\mathrm{X}$-ray constrained wavefunction fitting, the representation of properties on grids and the generation of a symmetry-generated crystallographic cluster of point charges for further theoretical calculations. At present, it is the only software that allows HAR-ELMO. lamaGOET is conceptually meant to be an interface for crystallographers who aim to work with Tonto for quantum-crystallographic applications and want to make the most of Tonto's vast functionality, or even extend it with external quantum-mechanical software. lamaGOET will be maintained and expanded in this direction. In this sense, the development of lamaGOET is different from recent HAR 
developments in Olex2 (Fugel et al., 2018) and from connections between the tsc format of nonspherical atomic form factors (Midgley et al., 2019) and HAR inside NoSpherA2 (Kleemiss, Dolomanov et al., 2021). These NoSpherA2-related developments make quantum-crystallographic refinements as simple and user friendly as possible and aim at a broad chemical audience, whereas lamaGOET remains a quantumcrystallographic tool centred around Tonto.

\section{Acknowledgements}

Open access funding enabled and organized by Projekt DEAL.

\section{Funding information}

SG thanks the German Research Foundation (Deutsche Forschungsgemeinschaft, DFG) for funding of an Emmy Noether project (GR 4451/1-1). AG thanks the French Research Agency (ANR) for financial support of the Young Investigator Project QuMacroRef (grant No. ANR-17-CE290005-01).

\section{References}

Allen, F. H. \& Bruno, I. J. (2010). Acta Cryst. B66, 380-386.

Bąk, J. M., Domagała, S., Hübschle, C., Jelsch, C., Dittrich, B. \& Dominiak, P. M. (2011). Acta Cryst. A67, 141-153.

Benabicha, F., Pichon-Pesme, V., Jelsch, C., Lecomte, C. \& Khmou, A. (2000). Acta Cryst. B56, 155-165.

Bergmann, J., Davidson, M., Oksanen, E., Ryde, U. \& Jayatilaka, D. (2020). IUCrJ, 7, 158-165.

Boese, R., Niederprüm, N., Bläser, D., Maulitz, A., Antipin, M. Y. \& Mallinson, P. R. (1997). J. Phys. Chem. B, 101, 5794-5799.

Bučinský, L., Jayatilaka, D. \& Grabowsky, S. (2016). J. Phys. Chem. A, 120, 6650-6669.

Bytheway, I., Chandler, G., Figgis, B. \& Jayatilaka, D. (2007). Acta Cryst. A63, 135-145.

Capelli, S. C., Bürgi, H.-B., Dittrich, B., Grabowsky, S. \& Jayatilaka, D. (2014). IUCrJ, 1, 361-379.

Cole, J. M. \& Hickstein, D. D. (2013). Phys. Rev. B, 88, 184105.

Compton, A. H. (1915). Nature, 95, 343-344.

Coppens, P. (1997). X-ray Charge Densities and Chemical Bonding. IUCr Texts on Crystallography, No. 4. Oxford Science Publications/ International Union of Crystallography.

Coppens, P. (2005). Angew. Chem. Int. Ed. 44, 6810-6811.

Dadda, N., Nassour, A., Guillot, B., Benali-Cherif, N. \& Jelsch, C. (2012). Acta Cryst. A68, 452-463.

Dawson, B. (1967). Proc. R. Soc. Lond. A, 298, 255-263.

Dennington, R. D., Keith, T. A. \& Millam, J. M. (2008). GaussView 5.0. Gaussian Inc., Wallingford, CT, USA.

Dittrich, B., Bond, C. S., Kalinowski, R., Spackman, M. A. \& Jayatilaka, D. (2010). CrystEngComm, 12, 2419-2423.

Dittrich, B., Hübschle, C. B., Messerschmidt, M., Kalinowski, R., Girnt, D. \& Luger, P. (2005). Acta Cryst. A61, 314-320.

Dittrich, B., Pfitzenreuter, S. \& Hübschle, C. B. (2012). Acta Cryst. A68, 110-116.

Dittrich, B., Sze, E., Holstein, J. J., Hübschle, C. B. \& Jayatilaka, D. (2012). Acta Cryst. A68, 435-442.

Dovesi, R., Erba, A., Orlando, R., Zicovich-Wilson, C., Civalleri, B., Maschio, L., Rérat, M., Casassa, S., Baima, J., Salustro, S. \& Kirtman, B. (2018). WIREs Comput. Mol. Sci. 8, e1360.

Ernst, M., Genoni, A. \& Macchi, P. (2020). J. Mol. Struct. 1209, 127975.
Frisch, M. J., Trucks, G. W., Schlegel, H. B., Scuseria, G. E., Robb, M. A., Cheeseman, J. R., Scalmani, G., Barone, V., Petersson, G. A., Nakatsuji, H., Li, X., Caricato, M., Marenich, A. V., Bloino, J., Janesko, B. G., Gomperts, R., Mennucci, B., Hratchian, H. P., Ortiz, J. V., Izmaylov, A. F., Sonnenberg, J. L., Williams-Young, D., Ding, F., Lipparini, F., Egidi, F., Goings, J., Peng, B., Petrone, A., Henderson, T., Ranasinghe, D., Zakrzewski, V. G., Gao, J., Rega, N., Zheng, G., Liang, W., Hada, M., Ehara, M., Toyota, K., Fukuda, R., Hasegawa, J., Ishida, M., Nakajima, T., Honda, Y., Kitao, O., Nakai, H., Vreven, T., Throssell, K., Montgomery, J. A. Jr, Peralta, J. E., Ogliaro, F., Bearpark, M. J., Heyd, J. J., Brothers, E. N., Kudin, K. N., Staroverov, V. N., Keith, T. A., Kobayashi, R., Normand, J., Raghavachari, K., Rendell, A. P., Burant, J. C., Iyengar, S. S., Tomasi, J., Cossi, M., Millam, J. M., Klene, M., Adamo, C., Cammi, R., Ochterski, J. W., Martin, R. L., Morokuma, K., Farkas, O., Foresman, J. B. \& Fox, D. J. (2016). Gaussian16 Revision C.01. Gaussian Inc., Wallingford, CT, USA.

Fugel, M., Jayatilaka, D., Hupf, E., Overgaard, J., Hathwar, V. R., Macchi, P., Turner, M. J., Howard, J. A. K., Dolomanov, O. V., Puschmann, H., Iversen, B. B., Bürgi, H.-B. \& Grabowsky, S. (2018). IUCrJ, 5, 32-44.

Furche, F., Ahlrichs, R., Hättig, C., Klopper, W., Sierka, M. \& Weigend, F. (2014). WIREs Comput. Mol. Sci. 4, 91-100.

Genoni, A., Bučinský, L., Claiser, N., Contreras-García, J., Dittrich, B., Dominiak, P. M., Espinosa, E., Gatti, C., Giannozzi, P., Gillet, J.-M., Jayatilaka, D., Macchi, P., Madsen, A. O., Massa, L., Matta, C. F., Merz, K. M., Nakashima, P. N. H., Ott, H., Ryde, U., Schwarz, K., Sierka, M. \& Grabowsky, S. (2018). Chem. Eur. J. 24, 10881-10905.

Genoni, A., Dos Santos, L. H. R., Meyer, B. \& Macchi, P. (2017). IUCrJ, 4, 136-146.

Giannozzi, P., Andreussi, O., Brumme, T., Bunau, O., Buongiorno Nardelli, M., Calandra, M., Car, R., Cavazzoni, C., Ceresoli, D., Cococcioni, M., Colonna, N., Carnimeo, I., Dal Corso, A., de Gironcoli, S., Delugas, P., DiStasio, R. A., Ferretti, A., Floris, A., Fratesi, G., Fugallo, G., Gebauer, R., Gerstmann, U., Giustino, F., Gorni, T., Jia, J., Kawamura, M., Ko, H., Kokalj, A., Küçükbenli, E., Lazzeri, M., Marsili, M., Marzari, N., Mauri, F., Nguyen, N. L., Nguyen, H.-V., Otero-de-la-Roza, A., Paulatto, L., Poncé, S., Rocca, D., Sabatini, R., Santra, B., Schlipf, M., Seitsonen, A. P., Smogunov, A., Timrov, I., Thonhauser, T., Umari, P., Vast, N., Wu, X. \& Baroni, S. (2017). J. Phys. Condens. Matter, 29, 465901.

Grabowsky, S., Genoni, A. \& Bürgi, H.-B. (2017). Chem. Sci. 8, 4159 4176.

Grabowsky, S., Genoni, A., Thomas, S. P. \& Jayatilaka, D. (2020). 21st Century Challenges in Chemical Crystallography II - Structural Correlations and Data Interpretation, Structure and Bonding, Vol. 186, edited by D. M. P. Mingos \& P. Rathby, pp 65-144. Cham: Springer Nature.

Grimwood, D. J. \& Jayatilaka, D. (2001). Acta Cryst. A57, 87-100.

Hansen, N. K. \& Coppens, P. (1978). Acta Cryst. A34, 909-921.

Hickstein, D. D., Cole, J. M., Turner, M. J. \& Jayatilaka, D. (2013). J. Chem. Phys. 139, 064108.

Hirshfeld, F. L. (1971). Acta Cryst. B27, 769-781.

Hirshfeld, F. L. (1977a). Theor. Chim. Acta, 44, 129-138.

Hirshfeld, F. L. (1977b). Isr. J. Chem. 16, 198-201.

Humphrey, W., Dalke, A. \& Schulten, K. (1996). J. Mol. Graph. 14, 33-38.

Jayatilaka, D. (1998). Phys. Rev. Lett. 80, 798-801.

Jayatilaka, D. \& Dittrich, B. (2008). Acta Cryst. A64, 383-393.

Jayatilaka, D. \& Grimwood, D. J. (2001). Acta Cryst. A57, 76-86.

Jayatilaka, D. \& Grimwood, D. J. (2003). Computational Science|ICCS 2003, edited by P. M. A. Sloot, D. Abramson, A. V. Bogdanov, Y. E. Gorbachev, J. J. Dongarra \& A. Y. Zomaya, pp. 142-151. Berlin, Heidelberg: Springer.

Jayatilaka, D., Munshi, P., Turner, M. J., Howard, J. A. \& Spackman, M. A. (2009). Phys. Chem. Chem. Phys. 11, 7209-7218.

Jelsch, C., Teeter, M. M., Lamzin, V., Pichon-Pesme, V., Blessing, R. H. \& Lecomte, C. (2000). Proc. Natl Acad. Sci. USA, 97, 3171-3176. 
Kleemiss, F., Dolomanov, O. V., Bodensteiner, M., Peyerimhoff, N., Midgley, L., Bourhis, L. J., Genoni, A., Malaspina, L. A., Jayatilaka, D., Spencer, J. L., White, F., Grundkötter-Stock, B., Steinhauer, S., Lentz, D., Puschmann, H. \& Grabowsky, S. (2021). Chem. Sci. 12, 1675-1692.

Kleemiss, F., Wieduwilt, E. K., Hupf, E., Shi, M. W., Stewart, S. G., Jayatilaka, D., Turner, M. J., Sugimoto, K., Nishibori, E., Schirmeister, T., Schmidt, T. C., Engels, B. \& Grabowsky, S. (2021). Chem. Eur. J. 27, 3407-3419.

Koritsanszky, T. S. \& Coppens, P. (2001). Chem. Rev. 101, 1583-1628.

Korlyukov, A. A. \& Nelyubina, Y. V. (2019). Russ. Chem. Rev. 88, 677-716.

Kurki-Suonio, K. (1968). Acta Cryst. A24, 379-390.

Macrae, C. F., Sovago, I., Cottrell, S. J., Galek, P. T. A., McCabe, P., Pidcock, E., Platings, M., Shields, G. P., Stevens, J. S., Towler, M. \& Wood, P. A. (2020). J. Appl. Cryst. 53, 226-235.

Malaspina, L. A. (2020). PhD thesis, https://doi.org/10.26092/elib/81.

Malaspina, L. A., Wieduwilt, E. K., Bergmann, J., Kleemiss, F., Meyer, B., Ruiz-López, M. F., Pal, R., Hupf, E., Beckmann, J., Piltz, R. O., Edwards, A. J., Grabowsky, S. \& Genoni, A. (2019). J. Phys. Chem. Lett. 10, 6973-6982.

Medvedev, M. G., Bushmarinov, I. S., Sun, J., Perdew, J. P. \& Lyssenko, K. A. (2017). Science, 355, 49-52.
Meyer, B. \& Genoni, A. (2018). J. Phys. Chem. A, 122, 8965-8981.

Meyer, B., Guillot, B., Ruiz-Lopez, M. F. \& Genoni, A. (2016). J. Chem. Theory Comput. 12, 1052-1067.

Meyer, B., Guillot, B., Ruiz-Lopez, M. F., Jelsch, C. \& Genoni, A. (2016). J. Chem. Theory Comput. 12, 1068-1081.

Midgley, L., Bourhis, L. J., Dolomanov, O., Peyerimhoff, N. \& Puschmann, H. (2019). arXiv:1911.08847.

Neese, F. (2012). WIREs Comput. Mol. Sci. 2, 73-78.

Sanjuan-Szklarz, W. F., Woińska, M., Domagała, S., Dominiak, P. M., Grabowsky, S., Jayatilaka, D., Gutmann, M. \& Woźniak, K. (2020). IUCrJ, 7, 920-933.

Sheldrick, G. M. (2008). Acta Cryst. A64, 112-122.

Stewart, R. F. (1976). Acta Cryst. A32, 565-574.

Whitten, A. E., Jayatilaka, D. \& Spackman, M. A. (2006). J. Chem. Phys. 125, 174505.

Woińska, M., Grabowsky, S., Dominiak, P. M., Woźniak, K. \& Jayatilaka, D. (2016). Sci. Adv. 2, e1600192.

Woińska, M., Jayatilaka, D., Dittrich, B., Flaig, R., Luger, P., Woźniak, K., Dominiak, P. M. \& Grabowsky, S. (2017). ChemPhysChem, 18, 3334-3351.

Zheng, M., Biczysko, M., Xu, Y., Moriarty, N. W., Kruse, H., Urzhumtsev, A., Waller, M. P. \& Afonine, P. V. (2020). Acta Cryst. D76, 41-50. 